\title{
Alcoolização de Nódulo Tiroidiano em Região Endêmica de Bócio Colóide
}

\begin{abstract}
RESUMO
A doença nodular tiroidiana é problema clínico comum, principalmente em regiões com carência de iodo. Esse estudo avaliou a eficácia da injeção percutânea de etanol (IPE) no tratamento de nódulos tiroidianos únicos, múltiplos, sólidos, císticos, de diferentes tamanhos com ou sem disfunção glandular, em região endêmica de bócio. Quarenta e dois pacientes com diagnóstico de bócio colóide ou hiperplasia nodular colóide, na punção biópsia aspirativa por agulha fina (PBAAF), foram selecionados para análise após terem sido submetidos a pelo menos duas IPEs. Os nódulos tiroidianos eram múltiplos (sólidos e ou císticos) em $52,4 \%$ dos pacientes, únicos e sólidos ou mistos em $35,7 \%$ e únicos e císticos em $11,9 \%$. A redução média dos nódulos após injeção de etanol foi de $58,2 \%$ para os únicos e $60,8 \%$ nos císticos. Nos nódulos múltiplos, avaliou-se a redução de todo o lobo tiroidiano, alcançando $52,4 \%$ de redução. Os efeitos colaterais registrados foram decorrentes apenas do desconforto na aplicação. Este estudo indica que a injeção percutânea de etanol (IPE), alcançando reduções médias de volume que variam de 49 a $60 \%$, é método seguro, eficaz e simples para o tratamento de nódulos tiroidianos benignos. (Arq Bras Endocrinol Metab 2007;51/6:1007-1012)
\end{abstract}

Descritores: Alcoolização; Tiróide; Bócio colóide; Injeção percutânea; Etanol

\section{ABSTRACT}

\begin{abstract}
Alcoholization in the Treatment of Thyroid Nodule in Colloid Goiter Endemic Region.

Thyroid nodular disease is a common clinical disorder mainly in iodine lacking regions. This study has evaluated the effectiveness of percutaneous ethanol injection (PEI) in the treatment of single, multiple, solid, and cystic thyroid nodules of different sizes with or without glandular dysfunction, in goiter endemic region. Forty-two patients with diagnosis of colloid goiter or colloid nodular hyperplasia in fine-needle aspiration (FNA) were selected for analysis, after having been submitted to at least two PEI. Thyroid nodules were multiple (solid and or cystic) in $52.4 \%$ of the patients, single and solid or mixed in $35.7 \%$, and single and cystic in $11.9 \%$. The mean reduction of nodules after ethanol injection was of $58.2 \%$ in the single and of $60.8 \%$ in the cystic ones. The reduction of the whole thyroid lobe was evaluated in the multiple nodules and it reached $52.4 \%$. The sideeffects were registered only as a consequence of the application discomfort. This study points out that the percutaneous ethanol injection reaching volume mean reductions, varying from $49 \%$ to $60 \%$, is a safe, effective, and simple method for the treatment of benign thyroid nodules. (Arq Bras Endocrinol Metab 2007;51/6:1007-1012)
\end{abstract}

Keywords: Alcoholization; Thyroid; Colloid goiter; Percutaneous injection; Ethanol artigo original

\author{
Marcus AurelHo Lima \\ TALES Alvarenga FAGUndes \\ CRISTIANE MENDES RAFFAELLI \\ BEATRIZ PIRES FERREIRA \\ ELISABETE MANTOVANI RESENDE \\ Elvi Cristina Rojas fonseca \\ MARIa de FÁtIMA Borges
}

Laboratório de Anatomia

Patológica e Citologia da

Associação de Combate ao

Câncer do Brasil Central (MAL \&

CMR), Universidade Federal do

Triângulo Mineiro (MAL, TAF, BPF, EMR, ECRF \& MFB), Uberaba, $M G$.

Recebido em 06/09/06

Revisado em 21/12/06

Aceito em 27/02/07 
$\mathrm{A}^{\mathrm{p}}$ PROXIMADAMENTE 4 A 7\% DA POPULAÇÃo geral têm nódulos tiroidianos palpáveis (1) e a região em que vivemos, o Triângulo Mineiro, é considerada área bocígena segundo relatório da Organização Mundial de Saúde $(2,3)$, apresentando grande número de tiroidopatias, especialmente aquelas relacionadas à carência de iodo, tais como bócio colóide e neoplasias. Dentre as tiroidopatias, a doença nodular por bócio colóide é a mais freqüente, tornando-se um problema clínico comum nessa região e com etiologias diversas (4).

A punção biópsia aspirativa por agulha fina (PBAAF) é o método de eleição para caracterização do nódulo como benigno ou maligno, porque é virtualmente isenta de complicações, tem baixo custo, fácil realização e, dentre os vários métodos propedêuticos, é o que fornece resultados mais satisfatórios (4).

O tratamento dos nódulos císticos através da aspiração do líquido e supressão com hormônios tiroidianos ou o tratamento esclerosante com outros agentes químicos como a tetraciclina tem obtido resultados insatisfatórios $(5,6)$. Atualmente, o etanol tem sido utilizado como o principal agente esclerosante nos nódulos císticos da tiróide (7).

Há várias vantagens da injeção percutânea de etanol (IPE) em comparação com o uso de radioiodo para tratamento de nódulos tiroidianos. Apesar de alguns resultados ainda controversos, parece tão efetivo quanto o radioiodo em induzir a cura parcial ou completa de nódulos autônomos, causando maior redução do volume do nódulo, sem recidiva e sem provocar hipotiroidismo $(8)$.

A injeção com etanol é recomendada como conduta terapêutica em uma lesão nodular da tiróide somente depois de afastada a hipótese de malignidade através de PBAAF (9).

A IPE pode ser uma alternativa aos pacientes portadores de lesões nodulares da tiróide que tenham indicação cirúrgica e que recusam esta forma de tratamento ou que apresentem alto risco cirúrgico (7).

Nesse estudo, será avaliada a eficácia da injeção percutânea de etanol como via alternativa, em região endêmica de bócio, para tratamento de nódulos tiroidianos únicos, múltiplos, sólidos, císticos, de diferentes tamanhos com ou sem disfunção glandular.

\section{MÉTODOS}

\section{Seleção dos casos}

Sessenta e um pacientes com nódulos tiroidianos foram selecionados no período de 2002 a 2004, com diagnóstico, na PBAAF, de bócio colóide ou hiperplasia nodular colóide. Antes da realização da PBAAF, os pacientes foram avaliados de acordo com sua história clínica e sinais físicos, função tiroidiana e ultra-sonografia, segundo rotina dos serviços de suas procedências. Dezenove pacientes foram excluídos, pois ao final desse trabalho ainda não haviam retornado para realizar a segunda injeção de etanol, permanecendo para análise de dados quarenta e dois pacientes.

$\mathrm{O}$ projeto de pesquisa foi submetido a avaliação pela comissão de Ética Médica da Universidade Federal do Triângulo Mineiro. Os pacientes foram encaminhados ao tratamento tendo conhecimento prévio do procedimento através de informativo, e todos assinaram o termo de consentimento.

\section{Técnica de aspiração}

A PBAAF foi realizada por médico citopatologista, como preconizada por Löwhagen (10). Do aspirado, de cada um dos nódulos, foram obtidos de 2 a 5 esfregaços, sendo secos ao ar e corados segundo a técnica de Romanovsky (11). O restante do aspirado, sob a forma de coágulo na seringa, foi imerso e fixado em álcool a 95\% e processado segundo a metodologia clássica de inclusão e execução dos cortes em parafina (12).

Os pacientes com bócio multinodular e nódulos confluentes foram aspirados em diferentes regiões da glândula, tentando atingir áreas ecogenicamente mais suspeitas de neoplasia, e, nos casos de bócio multinodular com nódulos separados por parênquima normal, todas as lesões foram aspiradas.

Os diagnósticos citopatológicos dos esfregaços foram baseados, fundamentalmente, na análise do parênquima tiroidiano e dos elementos adjacentes, segundo critérios já estabelecidos $(10,13,14)$. A análise histológica dos aspirados incluídos em parafina obedeceu aos critérios fundamentados na morfologia histopatológica clássica já bem determinada e conhecida.

\section{Técnica de injeção percutânea de etanol guiada por ultra-sonografia}

O paciente, depois de orientado quanto ao método, foi colocado na mesma posição da PBAAF ou de ultrasonografia. Recomendou-se o uso de analgésico uma hora antes do procedimento, aplicação de anestésico em gel tópico sobre a pele da região cervical anterior e, quando houve necessidade em virtude da maior sensibilidade do paciente, foi realizada sedação com Dormonid ${ }^{\circledR}$ (maleato de midazolam) em dose de 5 a $15 \mathrm{mg}$ por via endovenosa.

A ultra-sonografia foi realizada com aparelho da marca Toshiba Power Vision 6000, com transdutor linear com multifreqüência de 6,0 a 11,0 mHz. Para a injeção do etanol, foi utilizada agulha com comprimento de $2,5 \mathrm{~cm}$ e calibre de 0,6 mm (24 gauge) acoplada a seringa de injeção de insulina com volume de $1 \mathrm{ml}$, e o álcool foi injetado sempre lentamente. Antes da aplicação e nos intervalos, colocava-se bolsa gelada sobre a região cervical anterior.

O volume dos nódulos e lobos foi calculado multiplicando o eixo longitudinal pelo eixo transversal e pelo eixo ântero-posterior, e o produto multiplicado pela constante 0,52 . A ecografia foi realizada por médico imagenologista e a injeção de etanol, pelo citopatologista que executou a punção prévia. 
Nos nódulos sólidos, a quantidade total de etanol absoluto introduzida foi, no máximo, $20 \%$ do volume nodular, calculado previamente, até que eles se tornassem hiperecogênicos difusamente. $\mathrm{O}$ controle visual da disseminação do álcool intranodular, através da ultra-sonografia, foi utilizado para determinar o volume do etanol a ser aplicado em cada injeção. Quando o álcool preenchia todo o espaço nodular, o procedimento era interrompido.

As administrações do etanol foram realizadas em intervalos médios de seis meses. Nos retornos, realizava-se nova ultra-sonografia medindo o volume do nódulo e avaliando a necessidade de outra injeção.

A redução foi caracterizada em relação ao volume dos nódulos quando era único, e nos nódulos múltiplos e coalescentes foi considerado o volume inicial de todo o lobo e não de cada nódulo.

Quanto aos nódulos císticos, foi realizada aspiração quase total do conteúdo, deixando apenas líquido suficiente para encontrar a cavidade, seguida da aplicação definitiva de etanol absoluto na quantidade de $0,5 \mathrm{a} 1 \mathrm{ml}$. O procedimento era repetido após seis meses se ainda houvesse persistência do cisto.

Nos nódulos mistos, se a porção líquida era volumosa, aspirava-se, e o volume do nódulo era recalculado. Assim, o mesmo procedimento era aplicado aos nódulos sólidos.

\section{RESULTADOS}

A idade média do grupo examinada era 49,8 anos, com mediana de 49 anos. Quanto ao sexo, dois eram homens $(4,8 \%)$ e 40 mulheres $(95,2 \%)$.

Os nódulos tiroidianos eram múltiplos (sólidos e/ou císticos) em $52,4 \%$ dos pacientes, únicos e sóidos ou mistos em $35,7 \%$ e únicos e císticos em 11,9\% (figura 1 ).

Os resultados foram compilados daqueles 42 pacientes que retornaram para se submeter a, pelo menos, uma nova injeção de etanol, quando foi possível avaliar o resultado da terapêutica após a realização de nova ultra-sonografia.

A função tiroidiana dos pacientes encaminhados está apresentada na tabela 1 . Naqueles casos com hipertiroidismo laboratorial, 27,3\% apresentavam concentrações de hormônio tireoestimulante (TSH) abaixo do valor de referência com tiroxina livre (T4L) aumentada e $72,7 \%$ tinham apenas TSH suprimido.

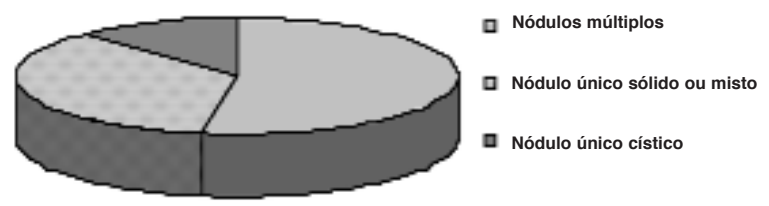

Figura 1. Distribuição dos casos quanto ao tipo de nódulo.
Apenas cinco pacientes (12\%) informaram o uso de terapia supressiva prévia com levotiroxina, com o objetivo de reduzir o tamanho do nódulo, e esse tratamento já tinha sido abandonado na época do início da injeção de etanol.

A redução média dos nódulos após injeção de etanol foi de $52,4 \%$ para os múltiplos e sólidos, $58,2 \%$ para os únicos e sólidos e $60,8 \%$ nos císticos e únicos, conforme está demonstrado na figura 2 .

\section{Nódulos múltiplos, sólidos e mistos}

Vinte e dois pacientes apresentavam nódulos múltiplos $(52,4 \%)$. Nesses casos, o tratamento tinha como objetivo a redução do volume do lobo tiroidiano. Desse modo, foram analisados 35 lobos e, destes, 11 pacientes sofreram injeção em apenas um lobo, nove pacientes nos dois lobos e 2 pacientes nos dois lobos e no istmo.

A redução variou conforme o número de aplicações, como mostra a figura 3 . O volume do lobo reduziu em $41 \%$ com uma aplicação em 14 pacientes, redução de $53 \%$ com duas aplicações em 13 pacientes, redução de $53 \%$ com três aplicações em 1 paciente e redução de $64,7 \%$ com quatro aplicações em 3 pacientes. A média foi de $49,2 \%$ de redução do volume com duas aplicações a intervalo de quatro meses.

\section{Nódulo único sólido}

Quinze pacientes (36\%) apresentavam nódulo único. A redução do volume variou de 55\% com uma aplicação em 7 pacientes, $61 \%$ com duas aplicações em 4 pacientes, $57 \%$ de redução com três aplicações em 3 pacientes e redução do volume do nódulo em $75 \%$ com quatro aplicações em 1 paciente. Estes resultados estão expressos na figura 4 .

A média foi de $58,2 \%$ de redução do volume inicial após duas aplicações com intervalo de 4,2 meses.

\section{Cistos}

Os cinco pacientes com cisto único (12\%) apresentaram redução média de $60,8 \%$ de seu volume inicial após média de 5,2 meses da primeira e única aplicação.

Tabela 1. Função tiroidiana em 42 pacientes estudados.

\begin{tabular}{lc}
\hline Função Tiroidiana & Número de pacientes (\%) \\
Normal & $26(62)$ \\
Hipertiroidismo & $11(26)$ \\
Hipotiroidismo & $1(02)$ \\
Indeterminada & $4(09)$ \\
\hline
\end{tabular}




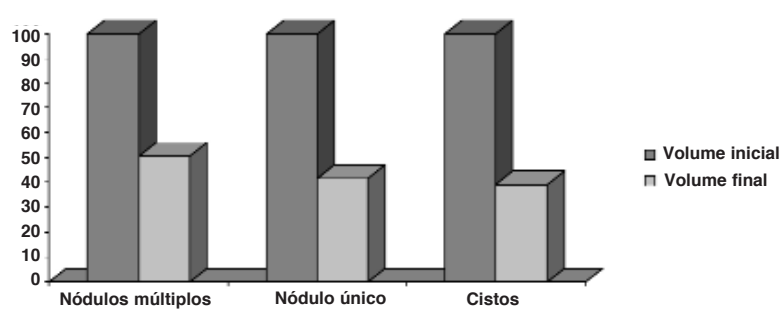

Figura 2. Percentual de redução do tamanho dos nódulos após tratamento com injeção percutânea de etanol.

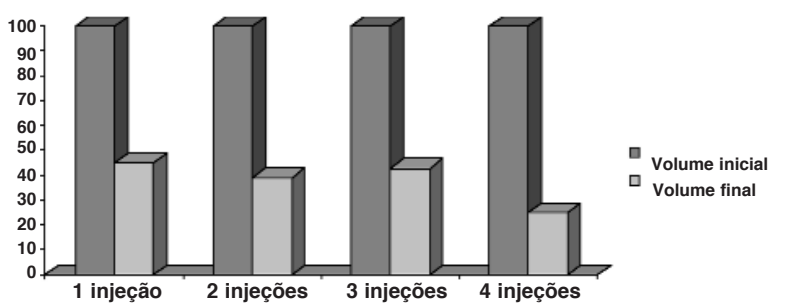

Figura 4. Percentual de redução dos nódulos únicos e sólidos após injeção de etanol.

\section{Número de injeções}

O número de aplicações variava de acordo com a resposta de cada caso, ou seja, até o volume dos nódulos atingir índices aceitáveis do ponto de vista estético ou quando a função tiroidiana era normalizada.

\section{Efeitos colaterais}

Durante a injeção, os pacientes queixavam-se de dor local, com irradiação para região auricular homolateral e/ou nas arcadas dentárias, que desaparecia logo após o término do procedimento, permanecendo maior sensibilidade à palpação na região cervical por doze a quarenta e oito horas. Alguns pacientes apresentaram hematoma (10 casos), disfonia transitória (dois casos) e/ou hipertermia (quatro casos).

\section{DISCUSSÃo}

Este estudo indica que a injeção percutânea de etanol (IPE), alcançando reduções médias de volume que variam de 49 a 60\%, é método seguro, eficaz e simples para o tratamento de nódulos tiroidianos benignos.

A redução do volume dos nódulos ocorreu com maior intensidade após aplicação da primeira injeção e as demais injeções resultam em reduções adicionais menos significativas (figura 2, 3, 4). Bennedbæk e cols. (15) mostraram diminuição média de $47 \%$ do volume

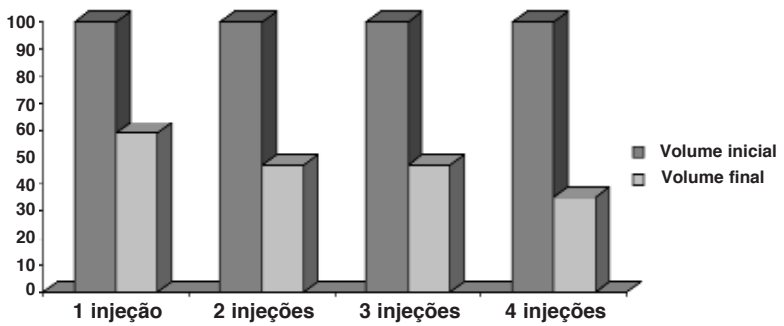

Figura 3. Percentual de redução dos lobos após injeção de etanol em bócios multinodulares.

de nódulos benignos frios após doze meses da aplicação de uma única injeção de etanol. Caraccio e cols. (16) demonstraram redução média de $70 \%$ do volume de nódulos benignos frios após múltiplas injeções de etanol ao longo de um ano. Bennedbæk e cols. mostraram, em estudo, que a diferença na redução do volume após uma ou três aplicações de álcool foi insignificante depois do período de seis meses (17).

A redução média dos nódulos após injeção de etanol, neste trabalho, foi de $\mathbf{5 2 , 4 \%}$ para os múltiplos, $58,2 \%$ para os únicos e $60,8 \%$ nos císticos (figura 2 ).

Levando em consideração as alterações ocasionadas pelo etanol no tecido tiroidiano como fibrose, necrose hemorrágica e inflamação (18), e que tais processos têm um período mínimo para se resolverem, é compreensível a diminuição contínua do volume dos nódulos observada até seis meses após uma aplicação. Dessa maneira, a realização das injeções com intervalos médios de seis meses, como demonstrado nesse trabalho, parece ser o mais adequado.

O mecanismo de ação do etanol absoluto, produzido a partir da cana de açúcar (Saccharum officinarum), parece estar relacionado à desidratação celular seguida por necrose por coagulação, trombose e oclusão vascular $(19,20)$. Nos cistos tiroidianos, a injeção de etanol leva à obstrução dos vasos nas paredes impedindo recidivas de hemorragias, e é comprovadamente mais eficaz do que a simples aspiração (21-23). Há descrições, também, de correlação entre ingestão de álcool e baixa prevalência de nódulo ou aumento do volume tiroidiano (bócio), provavelmente relacionados com efeito tóxico direto dessa substância na glândula $(24,25)$.

Em virtude de a IPE ser guiada por ultrasonografia, apenas o nódulo recebe o álcool e o tecido tiroidiano adjacente à injeção não apresenta alterações, o que explicaria o baixo índice de complicações clínicas precoces ou tardias (20). Outro ponto de interesse é a ausência de fibrose externa à cápsula da tiróide, não causando complicações para possível tiroidectomia posterior (7). 
Na literatura, é escassa a descrição de IPE em bócios multinodulares, principalmente não hiperfuncionantes. A utilização da injeção de etanol no presente trabalho foi realizada em casos de nódulos múltiplos após avaliação da benignidade pela PBAAF, tendo como princípio a punção de todos os nódulos que não eram confluentes no estudo ultrassonográfico. O resultado foi satisfatório nesses pacientes após a IPE, alcançando reduções médias de $52,4 \%$ no volume do lobo.

A IPE guiada por ultra-sonografia é tratamento efetivo e seguro para diversas lesões benignas e malignas de diferentes órgãos $(7,19,26-29)$. Na década de 80 , foi utilizada com o objetivo de tratar cistos renais, adenoma de paratiróide e pequenos carcinomas hepatocelulares (19,30,31). Em 1990, Livraghi e cols. (32) propuseram o seu uso para o tratamento de nódulos tiroidianos autônomos, obtendo regressão anatômica das lesões e cura da disfunção glandular. $\mathrm{Na}$ última década, vários grupos, especialmente europeus, têm confirmado os bons resultados desta terapia, tanto em nódulos tóxicos $(6,33,34)$ como atóxicos e císticos $(7,22,36)$

Um seguimento de cinco anos nos pacientes com nódulos autônomos da tiróide tratados pelo método demonstrou eficiência e segurança (34). A cura completa, definida como normalização dos hormônios tiroidianos e do TSH, assim como normalização cintilográfica do tecido tiroidiano extranodular, foi obtida em $68 \%$ a $100 \%$ dos nódulos pré-tóxicos e de 50 a $89 \%$ dos nódulos tóxicos (6). Dos 42 pacientes analisados neste estudo, onze apresentavam hiperfunção tiroidiana (tabela 1). Nestes casos, os hormônios tiroidianos normalizaram-se após a alcoolização.

Os cistos tiroidianos puros correspondem a pequenas imagens císticas de alguns milímetros de diâmetro, anecóicas e com reforço acústico posterior, sendo raramente malignas. Estas lesões, de difícil diagnóstico no passado, são hoje facilmente identificadas ao exame ultrassonográfico da tiróide (7) e respondem favoravelmente a injeção de etanol como demonstrado na literatura (21). Esta série mostra uma redução de 60,8\% do volume após uma injeção.

Excetuando-se as pequenas imagens císticas simples, as lesões nodulares com componente líquido devem ser submetidas a PBAAF dirigida pela ultra-sonografia. Qualquer conduta terapêutica em uma lesão nodular da tiróide deve ser considerada somente depois de afastada a hipótese de malignidade. Para isso, o tratamento será iniciado após o diagnóstico de bócio colóide através da PBAAF e, se possível, com inclusão em parafina do aspirado para aumentar a acurácia diagnóstica do método (35).
A IPE é uma alternativa eficaz para o tratamento de nódulos e cistos tiroidianos benignos como demonstrado no presente estudo. É imprescindível que a hipótese de malignidade seja afastada por PBAAF antes de ter início esta modalidade terapêutica. Como nas outras formas de tratamento, é necessário o seguimento posterior desses pacientes.

\section{REFERÊNCIAS}

1. Gharib $H$. Current evaluation of thyroid nodules. Trends Endocrinol Metab 1994;5:365-9.

2. Dunn JT, Van Der Haar FA. A practical guide to the correlation of iodine deficiency. International Council for Control of lodine Deficiency Disorders. Netherlands: Unicef-World Health Organization, 1990.

3. Hetzel BS. The prevention and control of iodine deficiency disorders. Administrative Commitee or Coordination Subcommitee on Nutrition policy discussion paper. New York: United Nations, 1988.

4. Hegedüs L, Bonnema SJ, Bennedbæk FN. Management of simple nodular goiter: current status and future perspectives. Endocr Rev 2003;24:102-32.

5. Hegedus L, Hansen JM, Karstrup S, Torp-Pedersen S, Juul N. Tetracycline for sclerosis of thyroid cysts. A randomized study. Arch Intern Med 1988;148:1116-8.

6. Bennedbæk FN, Karstrup S, Hegedus L. Percutaneous ethanol injection therapy in the treatment of thyroid and parathyroid diseases. Eur J Endocrinol 1997;136:240-50.

7. Camargo RYA, Tomimori EK. Injeção percutânea de etanol dirigida pelo ultra-som no tratamento dos nódulos tireóideos. Arq Bras Endocrinol Metab 1998;42:292-5.

8. Ferrari C, Reschini E, Paraccchi A. Treatment of the autonomous thyroid nodule: a review. Eur $\mathbf{J}$ Endocrinol 1996; 135:383-90.

9. Hegedus, L. The thyroid nodule. N Engl J Med 2004;351:1764-71.

10. Löwhagen T, Granberg P-O, Lundell G, Skinnari P, Sundblad $R$, Willems JS. Aspiration biopsy cytology (ABC) in nodules of the thyroid gland suspected to be malignant. Surg Clin North Am 1979;59:3-18.

11. Linsk JA, Franzen S. Intoduction. In: Linsk JA, Franzen S. Clinical Aspiration Cytology. Philadelphia: J.B. Lippincott, 1989. pp. 1-15.

12. Michalany J. Fixação. In: Michalany J (ed). Técnica Histológica em Anatomia Patológica. São Paulo: E.P.U., 1980. pp. $40-115$.

13. Chu EW, Hanson TA, Goldman JM, Robbins J. Study of cells in fine needle aspirations of the thyroid gland. Acta Cytol 1979;23:309-14.

14. Davidson HG, Campora RG, Thyroid. In: Bibbo M (ed). Comprehensive Cytopathology. Philadelphia: W. B. Saunders, 1991. pp. 649-70.

15. Bennedbæk FN, Nielsen LK, Hegedüs L. Effect of percutaneous ethanol injection therapy vs. suppressive doses of Lthyroxine on benign solitary solid cold thyroid nodules: a randomised trial. J Clin Endocrinol Metab 1998;83:30-5.

16. Caraccio N, Goletti O, Lippolis PV, Casolaro A, Cavina E, Miccoli $P$, et al. Is percutaneous ethanol injection a useful alternative for the treatment of the cold benign thyroid nodule? Five years' experience. Thyroid 1997;7:699-704.

17. Bennedbæk FN, Hegedüs L. Percutaneous ethanol injection therapy in benign solitary solid cold thyroid nodules: a randomized trial comparing one injection with three injections. Thyroid 1999;9:225-33.

18. Pomorski L, Bartos $\mathrm{M}$. Histologic changes in thyroid nodules after percutaneous ethanol injection in patients subsequently operated on due to new focal thyroid lesions. APMIS 2002;110:172-6. 
19. Solbiati L, Giangrande A, De Pra L, Bellotti E, Cantú P, Ravetto $C$. Percutaneous ethanol injection of parathyroid tumors under US guidance treatment for secondary hyperparathyroidism. Radiology 1985;155:607-10.

20. Crescenzi A, Papini E, Pacella CM, Rinaldi R, Panunzi C, Petrucci L, et al. Morphological changes in a hyperfunctioning thyroid adenoma after percutaneous ethanol injection: Histological, enzymatic and sub-microscopical alterations. $\mathbf{J}$ Endocrinol Invest 1996;19:371-6.

21. Verde G, Papini E, Pacella M, Galloti C, Delpiano S, Strada S, et al. Ultrasound guided percutaneous ethanol injection in the treatment of cystic thyroid nodules. Clin Endocrinol 1994;41:719-24.

22. Bennedbæk FN, Hegedüs L. Treatment of recurrent thyroid cysts with ethanol: a random double-blind controlled trial. $\mathbf{J}$ Clin Endocrinol Metab 2003;88:5773-7.

23. Guglielmi R, Pacella CM, Bianchini A, Bizzarri G, Rinaldi R, Graziano FM, et al. Percutaneous ethanol injection treatment in benign thyroid lesions: role and efficacy. Thyroid 2004:14:125-31.

24. Knudsen N, Bülow I, Laurberg P, Perrild H, Ovesen L, Jorgensen $T$. Alcohol consumption is associated with reduced prevalence of goitre and solitary thyroid nodules. Clin Endocrinol 2001;55:41-6.

25. Hegedüs L, Rasmussen N, Ravn V, Kastrup J, Krogsgaard K, Aldershvile J. Independent effects of liver disease and chronic alcoholism on thyroid function and size: the possibility of a toxic effect of alcohol on the thyroid gland. Metabolism 1988;37:229-33.

26. Bean J. Renal cysts: treatment with alcohol. Radiology $1981 ; 138: 329-31$.

27. Bean J, Rodan BA. Hepatic cysts: treatment with alcohol. Am J Radiol 1985;144:237-41.

28. Karstrup S, Holm HH, Torp-Pedersen S, Hegedüs L. Ultrasonically guided percutaneous inactivation of parathyroid tumors. Br J Radiol 1987;60:667-70.

29. Livraghi T, Bolondi L, Lazzaroni S, Marin G, Morabito A, Rapaccini GL, et al. Percutaneous ethanol injection in the treatment of hepatocellular carcinoma cirrhosis. Cancer 1992;69:925-9.
30. Charboneau JW, Hay ID, Van Heerden JA. Persistent primary hyperparathyroidism: successful ultrasound-guided percutaneous ethanol ablation of an occult adenoma. Mayo Clin Proc 1988;63:913-7.

31. Livraghi T, Festi D, Monti F, Salmi A, Vettori C. US-guided percutaneous alcohol injection of small hepatic and abdominal tumors. Radiology 1986;161:309-12.

32. Livraghi T, Paracchi A, Ferrari C, Bergonzi M, Garavaglia G, Raineri $P$, et al. Treatment of autonomous thyroid nodules with percutaneous ethanol injection: preliminary results. Radiology 1990;75:827-9.

33. Livraghi T, Paracchi A, Ferrari C, Reschini E, Macchi RM, Bonifacino A. Treatment of autonomous thyroid nodules with percutaneous ethanol injection: 4 year experience. Radiology 1994; 190:529-33.

34. Monzani F, Caraccio N, Goletti O, Lippolis PV, Casolaro A Guerra PD, et al. Five-year follow-up of percutaneous ethanol injection for the treatment of hyperfunctioning thyroid nodules: a study of 117 patients. Clin Endocrinol 1997:46:9-15.

35. Lima MA, Jorge BH, Barros MAE, Lopes ER. A utilidade da inclusão em parafina como técnica complementar no diagnóstico dos aspirados de tiróide. Rev Ass Med Brasil 1994;40(4):236-42.

36. Bianchini EX, Ikejiri ES, Mamone MC, Paiva ER, Maciel RMB, Furlanetto RP. Injeção percutânea de etanol no tratamento de nódulos tiroidianos sólidos, císticos e autônomos. Arq Bras Endocrinol Metab 2003;47:543-51.

\section{Endereço para correspondência:}

Marcus Aurelho Lima

Endocrinologia - Hospital Escola da Faculdade de Medicina

Universidade Federal do Triângulo Mineiro

Rua Getúlio Guaritá 130

38025-440 Uberaba, MG

Fax: (34) 3318-9903

E-mail: lima@mednet.com.br 